PROCEEDINGS OF THE

AMERICAN MATHEMATICAL SOCIETY

Volume 137, Number 8, August 2009, Pages 2767-2772

S 0002-9939(09)09846-3

Article electronically published on March 5, 2009

\title{
PROPER PRODUCTS
}

\author{
OTMAR SPINAS
}

(Communicated by Julia Knight)

\begin{abstract}
We show that the natural amoeba forcing associated with Laver forcing $\mathbb{L}$, Miller forcing $\mathbb{M}$ respectively, is proper. As a corollary we obtain that every finite power of $\mathbb{L}$, respectively $\mathbb{M}$, is proper.
\end{abstract}

\section{INTRODUCTION AND TERMINOLOGY}

Laver forcing $\mathbb{L}$ and Miller forcing $\mathbb{M}$ are two of the most important forcing notions of set theory of the reals. Laver forcing was introduced by Richard Laver in his famous paper 3] from 1976 in order to prove the consistency of the Borel conjecture. It consists of all trees $p \subseteq \omega^{<\omega}$ that have a stem, i.e. a shortest splitnode, denoted $\operatorname{stem}(p)$, and the property that every node extending $\operatorname{stem}(p)$ is an infinite splitnode, i.e. has infinitely many immediate successor nodes. The order is inclusion. Miller forcing $\mathbb{M}$ was introduced by Arnie Miller in [4. It consists of all nonempty trees $p \subseteq \omega^{<\omega}$ such that every $\sigma \in p$ has an extension $\tau \in p$ that is an infinite splitnode. It is convenient and irrelevant for the forcing properties of $\mathbb{M}$ to assume (and hence we shall do so) that $p$ has only infinite splitnodes. The set of all these will be denoted by $\operatorname{Split}(p)$. Again, the order on $\mathbb{M}$ is inclusion.

Laver's proof in 3 that $\mathbb{L}$ does not collapse $\aleph_{1}$ was certainly a crucial step towards Baumgartner's notion of Axiom $A$ forcing 1 and later of Shelah's proper forcing [5]. The key element of Laver's proof is the fact that for every $p \in \mathbb{L}$ and every dense open set $D \subseteq \mathbb{L}$ there exist $q \in \mathbb{L}$ and a countable $F \subseteq D$ such that $q \leqslant{ }^{0} p$, i.e. $q \leqslant p$ and $\operatorname{stem}(p)=\operatorname{stem}(q)$, and $[q] \subseteq \bigcup_{r \in F}[r]$. The analogous fact trivially holds for $\mathbb{M}$. Using this fact one can perform what is nowadays called a simple fusion argument to take care of countably many dense sets and thus show that $\mathbb{L}$ and $\mathbb{M}$ are proper.

Over the past decade, for various reasons, the finite powers of $\mathbb{L}$ and $\mathbb{M}$ and of related classical forcing notions have become the focus of several set theorists' attention (see e.g. [6], 9], 2], 8]). By a result in [9] (see 2]) it follows that $\mathbb{M}^{3}$ adds a Cohen real. On the other hand, in [8] it was shown that $\mathbb{M}^{2}$ is proper, does not add a Cohen real and shares a number of nice Ramsey-theoretic properties. It is easy to see that $\mathbb{L}^{2}$ does add a Cohen real (see Remark 2.17). Thus $\mathbb{M}^{3}$ and $\mathbb{L}^{2}$ (and any higher power) were considered uninteresting from the forcing point

Received by the editors December 10, 2007, and, in revised form, March 28, 2008, and December 5,2008 .

2000 Mathematics Subject Classification. Primary 03E05.

The author is partially supported by DFG grant SP 683/1-2.

(C)2009 American Mathematical Society Reverts to public domain 28 years from publication 
of view, though the puzzling question remained (and has been asked by Brendle, Zapletal, this author and possibly others) whether they are proper at all.

It is well-known that a countable (full-support) product of Cohen forcing collapses $\aleph_{1}$; thus $\mathbb{L}^{\omega}$ and $\mathbb{M}^{\omega}$ do as well. A consequence of our main result here will be that every finite product of the form $\mathbb{L}^{n} \times \mathbb{M}^{m}$ is proper. This result holds even more generally, as we could add finitely many factors of virtually every classical tree forcing. However, it is an open problem to find a reasonably large and nicely definable class of proper forcings that is closed under taking finite products. Our main result is that the natural amoeba forcings $\mathbb{A}(\mathbb{L})$ and $\mathbb{A}(\mathbb{M})$ associated with $\mathbb{L}$ and $\mathbb{M}$ are proper. Moreover we show that $\mathbb{L}^{n}$ is a complete subforcing of $\mathbb{A}(\mathbb{L})$ for every $n$, similarly for $\mathbb{M}$. In order to define $\mathbb{A}(\mathbb{L}), \mathbb{A}(\mathbb{M})$ respectively, let us first define the natural well-ordering of $\omega^{<\omega}$ : For $\sigma, \tau \in \omega^{<\omega}$ we let $\sigma \prec \tau$ iff $\max \{|\sigma|, \max (\operatorname{ran}(\sigma))\}<\max \{|\tau|, \max (\operatorname{ran}(\tau))\}$, or if equality holds, then either $|\sigma|<|\tau|$ or $|\sigma|=|\tau|$ and $\sigma$ precedes $\tau$ lexicographically. Clearly $\left(\omega^{<\omega}, \prec\right)$ has order-type $\omega$, so let $S: \omega \rightarrow \omega^{<\omega}$ be the order-isomorphism.

Given $p \in \mathbb{L}$ there exists a natural isomorphism $\pi$ between $\left(\omega^{<\omega}, \subseteq\right)$ and $(\operatorname{Split}(p)$, $\subseteq)$, so that $\pi(\emptyset)=\operatorname{stem}(p)$ and for every $\sigma \in \omega^{<\omega}$ and $\tau \in \operatorname{Split}(p)$ with $\pi(\sigma)=\tau$, for every $i<j<\omega$ with $\tau^{\wedge} i, \tau^{\wedge} j \in p$ there exist $m<n<\omega$ such that $\pi\left(\sigma^{\curlyvee} m\right)=\tau^{\wedge} i$ and $\pi\left(\sigma^{\wedge} n\right)=\tau^{\curlyvee} j$. By an initial segment of $\operatorname{Split}(p)$ we mean a finite sequence of the form $\langle\pi(S(i)): i<n\rangle$ for some $n<\omega$. To understand the main points of this paper it is important to notice that generally and typically such an initial segment of $\operatorname{Split}(p)$ is not the same thing as an initial segment of (Split $(p), \prec)$, where $\prec$ is the well-order of $\omega^{<\omega}$ defined above, restricted to $\operatorname{Split}(p)$. If an initial segment of $\operatorname{Split}(p)$ is also an initial segment of $(\operatorname{Split}(p), \prec)$, we call it increasing. Clearly for every $p \in \mathbb{L}$ there exists $q \in \mathbb{L}$ such that $q \leqslant 0$ and every initial segment of $\operatorname{Split}(q)$ is increasing. But the set of these $q$ 's is nowhere open. Now $\mathbb{A}(\mathbb{L})$ consists of all $(F, p)$ such that $p \in \mathbb{L}$ and $F$ is an initial segment of $\operatorname{Split}(p)$ (note that every initial segment of $\operatorname{Split}(p)$ is determined by its range). The order is defined by letting $(F, p) \leqslant(H, q)$ iff $F \supseteq H$ and $p \subseteq q$. If $G$ is an $\mathbb{A}(\mathbb{L})$-generic filter over $\mathbb{V}$, by genericity $p_{G}:=\bigcup\{F: \exists p(F, p) \in G\}$ is a Laver tree, and by Laver's key observation mentioned above (for his proof that $\mathbb{L}$ does not collapse $\aleph_{1}$ ) we see that every branch $x \in\left[p_{G}\right]$ is a Laver real over $\mathbb{V}$.

By [8, Main Lemma 2.2] the analogue of this key observation holds for $\mathbb{M}^{2}$ : Given $(p, q) \in \mathbb{M}^{2}$ and $D \subseteq \mathbb{M}^{2}$ dense open, there exist $\left(p^{\prime}, q^{\prime}\right) \in \mathbb{M}^{2},\left(p^{\prime}, q^{\prime}\right) \leqslant(p, q)$ (i.e. $p^{\prime} \leqslant{ }^{0} p$ and $q^{\prime} \leqslant{ }^{0} q$ ) and some countable $F \subseteq D$ such that $\left[p^{\prime}\right] \times\left[q^{\prime}\right] \subseteq$ $\bigcup_{(u, v) \in F}[u] \times[v]$. However this analogue fails for $\mathbb{L}^{2}$ : For every $(p, q) \in \mathbb{L}^{2}$ there exist $(x, y) \in[p] \times[q]$ such that $x(n)<y(n)$ for every $n \geqslant \max \{|\operatorname{stem}(p)|,|\operatorname{stem}(q)|\}$. But on the other hand, for every $k$ the set of $(p, q) \in \mathbb{L}^{2}$ such that every $(x, y) \in[p] \times[q]$ oscillates at least $k$ times (i.e. there are $n_{0}<\ldots<n_{k-1}$ such that $x\left(n_{i}\right)<y\left(n_{i}\right)$ and $x\left(n_{i}+1\right)>y\left(n_{i}+1\right)$ for every $\left.i<k\right)$ is open dense. We conclude that not every pair $(x, y) \in\left[p_{G}\right]^{2}$ with $x \neq y$ is $\mathbb{L}^{2}$-generic (see Remark 2.17).

To my knowledge, this is the first time that these most natural amoeba forcings $\mathbb{A}(\mathbb{L})$ and $\mathbb{A}(\mathbb{M})$ have been investigated. In [7] we worked with a different kind of amoeba forcing, where we required that the conditions $(F, p)$ have the property that $F$ is an increasing initial segment of $\operatorname{Split}(p)$. These are not complete subforcings of the natural ones considered here, though they are nice to work with, as they have much stronger forcing properties (e.g. don't add Cohen reals). 
It is quite easy to see (see Remark 2.3) that $\mathbb{A}(\mathbb{M})$ is essentially isomorphic to any of its finite powers; hence these are proper as well. Zapletal has trivialized our result that $\mathbb{L}^{n}$ is proper by showing that $\mathbb{L}$ can be completely embedded into an iteration of a $\sigma$-closed and a $\sigma$-centered forcing. Moreover he noticed that any finite product of such iterations is again of this form, hence proper. However, his observation does not seem to be true for $\mathbb{M}$.

\section{Oscillating trees}

Here we prove our main results for Miller forcing. For $\mathbb{L}$, definitions and proofs carry over naturally.

Definition 2.1. We define $\mathbb{A}(\mathbb{M})$, amoeba forcing associated with Miller forcing $\mathbb{M}$, to consist of all pairs $(F, p)$ such that $p \in \mathbb{M}$ and $F$ is a finite set of splitnodes of $p$. The order is defined by letting $(F, p) \leqslant(H, q)$ iff $p \subseteq q$ and $F \supseteq H$. If $G$ is an $\mathbb{A}(\mathbb{M})$-generic filter over some model of $Z F C$, then $S_{G}:=\bigcup\{F: \exists p(F, p) \in G\}$ uniquely determines a tree $p_{G} \in \mathbb{M}$ so that $S_{G}$ is the set of splitnodes of $p_{G}$.

Remark 2.2. In Definition 2.1 we used our convention from the introduction that our Miller trees only have infinite splitnodes. This is necessary to conclude that $p_{G} \in \mathbb{M}$. We could work slightly more generally and remove this convention. But in this case we have to define the order on $\mathbb{A}(\mathbb{M})$ so that $(F, p) \leqslant(H, q)$ iff $F \supseteq H, p \subseteq q$ and every element of $H$ that is an infinite splitnode of $q$ is an infinite splitnode of $p$. The properness of this forcing is proved as in Theorem 2.12 below. The only place where we use this more general definition is in the next remark.

Remark 2.3. Here we use the definition of $\mathbb{A}(\mathbb{M})$ in Remark 2.2. We claim that $\mathbb{A}(\mathbb{M})^{2}$ with the product order (and similarly every finite power of $\mathbb{A}(\mathbb{M})$ ) is isomorphic to $\mathbb{A}(\mathbb{M})$ below some of its conditions. Given any set $X$ of finite sequences, we let $a^{\frown} X=\left\{\tau: \exists \sigma \in X \tau=a^{\frown} \sigma\right\}$. Now given $((F, p),(H, q)) \in \mathbb{A}(\mathbb{M})^{2}$ we map it to $\left(0^{\frown} F \cup 1^{\frown} H \cup\{\emptyset\}, 0^{\frown} p \cup 1^{\frown} q\right)$. This is an isomorphism between $\mathbb{A}(\mathbb{M})^{2}$ and $\mathbb{A}(\mathbb{M})$ below the condition $\left(\{\emptyset\}, 0^{\frown} \omega^{<\omega} \cup 1^{\frown} \omega^{<\omega}\right)$. If we care only for complete embeddability, we do not need any finite splittings.

Fact 2.4. (a) $\mathbb{A}(\mathbb{L})^{n}$, and hence also $\mathbb{L}^{n}$, is a complete subforcing of $\mathbb{A}(\mathbb{L})$.

(b) $\mathbb{A}(\mathbb{M})^{n}$, and hence also $\mathbb{M}^{n}$, is a complete subforcing of $\mathbb{A}(\mathbb{M})$.

Proof. Given $\left\langle\left(F_{i}, p_{i}\right): i<n\right\rangle \in \mathbb{A}(\mathbb{M})^{n}$, map it to the condition $(H, q) \in \mathbb{A}(\mathbb{M})$, where $H=\{\emptyset\} \cup \bigcup_{i<n} i^{\urcorner} F_{i}$ and $q=\bigcup_{i<n} i^{\urcorner} p_{i} \cup \bigcup_{i \geqslant n} i^{\urcorner} \omega^{<\omega}$, similarly for $\mathbb{L}$.

Remark 2.5. Let $\mathbb{A}(\mathbb{M})^{\prime}$ consist of those $(F, p) \in \mathbb{A}(\mathbb{M})$ such that the following hold:

1) $F \neq \emptyset$;

2) there exists an isomorphism $\pi$ between $(\{S(i): i<n\}, \subseteq)$ and $(F, \subseteq)$ (hence $n=|F|)$ that respects the "moving right order" of all successor nodes of any element of $F$; i.e. given $\sigma \in F$ and two immediate successor nodes $\tau, \tau^{\prime} \in F$ of $\sigma$ such that $\tau(|\sigma|)<\tau^{\prime}(|\sigma|)$, then $\pi^{-1}(\tau) \prec \pi^{-1}\left(\tau^{\prime}\right)$;

3) no member of $F$ has an initial segment that is a splitnode of $p$ but not a member of $F$.

Such an $F$ is called an initial segment of $\operatorname{Split}(p)$. Then $\mathbb{A}(\mathbb{M})^{\prime}$ is a dense subset of $\mathbb{A}(\mathbb{M})$. Therefore we shall henceforth mean $\mathbb{A}(\mathbb{M})^{\prime}$ when we write $\mathbb{A}(\mathbb{M})$. Note that from property 2 it does not follow that $i<j<n$ implies $\pi(S(i)) \prec \pi(S(j))$. In the case that $F$ does have this property we call it increasing. It should be clear 
that forcing with all $(F, p) \in \mathbb{A}(\mathbb{M})$ such that $F$ is increasing is not dense in $\mathbb{A}(\mathbb{M})$, and actually is not even a complete subforcing of $\mathbb{A}(\mathbb{M})$. This has been studied in [7.

Definition 2.6. Suppose that $(F, p) \in \mathbb{A}(\mathbb{M})$ and $H \subseteq F$ such that stem $(p) \in H$ and $(H, q) \in \mathbb{A}(\mathbb{M})$ for some $q \leqslant p$ (note that according to Remark 2.5 $(H, p)$ need not be a member of $\mathbb{A}(\mathbb{M}))$. Let $p(H)$ consist of all $\sigma \in p$ such that for all $\tau \in H$, either $\sigma \subseteq \tau$ or the longest common initial segment of $\sigma$ and $\tau$ belongs to $H$. Note that $(H, \bar{p}(H)) \in \mathbb{A}(\mathbb{M})$ and $q \leqslant p(H)$ for every $q$ such that $(H, q) \in \mathbb{A}(\mathbb{M})$.

Definition 2.7. Let $(F, p) \in \mathbb{A}(\mathbb{M})$ and let $\pi$ be the isomorphism between $(\{S(i)$ : $i<n\}, \subseteq$ ) and $(F, \subseteq)$ (see Remark 2.5). Then we say that $\pi(S(n-1)$ ) is the last element of $F$. Let $i<n-1$ (if it exists) be such that $\pi(S(n-1))$ is the successor splitnode of $\pi(S(i))$ in $p$ and let $k=|\pi(S(i))|$. We say that $F$ is leading if $\pi(S(n-1))(k)>\tau(j)$ for every $\tau \in F \backslash\{\pi(S(n-1))\}$ and $j<|\tau|$. If $F$ is leading, we say that $p$ oscillates at $F$ if for every $x \in[p]$ the following holds: If $\sigma$ is the longest member of $F$ such that $\sigma \subseteq x$, then $x(|\sigma|)>\pi(S(n-1))(j)$ for every $j<|\pi(S(n-1))|$ (where $n$ is as above).

The following is clear:

Fact 2.8. For every $n$, the set of all $(F, p) \in \mathbb{A}(\mathbb{M})$ such that $|F| \geqslant n, F$ is leading, the last element of $F$ has length at least $n$, and $p$ oscillates at $F$ is dense.

Definition 2.9. Let $(F, p) \in \mathbb{A}(\mathbb{M})$ such that $F$ is leading, and let $\tau$ be the last element of $F$. We define $O T(p, F)$, the oscillating subtree of $p$ with respect to $F$, to be the set of all $\sigma \in p$ such that either $\sigma$ is an initial segment of an element of $F$ or else, if $\varsigma \in F$ is the longest proper initial segment of $\sigma$, then $\sigma(|\varsigma|)>\tau(j)$ for every $j<|\tau|$.

Main Lemma 2.10. Let $(F, p) \in \mathbb{A}(\mathbb{M})$ and let $D \subseteq \mathbb{A}(\mathbb{M})$ be dense and open. There exists $(F, q) \in \mathbb{A}(\mathbb{M})$ such that $(F, q) \leqslant(F, p)$ and for every leading $H \subseteq$ Split $(q)$ with $F \subseteq H$ the following holds: If $(H, q) \in \mathbb{A}(\mathbb{M})$ and there exists $\left(H, q^{\prime}\right) \in$ $\mathbb{A}(\mathbb{M})$ such that $\left(H, q^{\prime}\right) \leqslant(H, q)$ and $\left(H, q^{\prime}\right) \in D$, then $(H, O T(q, H)) \in D$.

Proof. In order to simplify our notation we assume that $F=\{\operatorname{stem}(p)\}$. The general case is handled completely analogously. We are going to define sequences $\left\langle q_{k}: k<\omega\right\rangle$ in $\mathbb{M}$ and $\left\langle\mu_{k}: k<\omega\right\rangle$ in $\omega^{<\omega}$ such that $q_{0}=p, q_{k+1} \leqslant^{0} q_{k}, \mu_{0}=$ $\operatorname{stem}\left(q_{0}\right)$ and $\left\langle\mu_{i}: i \leqslant k\right\rangle$ is leading, increasing and an initial segment of the splitnodes of $q_{k}$. We intend to let $q$ be the tree generated by all the $\mu_{k}$. Suppose that we have obtained $\left\langle q_{i}: i \leqslant k\right\rangle$ and $\left\langle\mu_{i}: i \leqslant k\right\rangle$. Let $\mathcal{H}$ be the set of all $H \subseteq\left\{\mu_{i}\right.$ : $i \leqslant k\}$ such that $\mu_{k} \in H, q_{k}(H)$ is well defined, and hence $\left(H, q_{k}(H)\right) \in \mathbb{A}(\mathbb{M})$, and $H$ is leading. Then clearly $\mu_{k}$ is the last element of $H$. Now we claim that there exists $q_{k+1} \leqslant q_{k}$ such that $\left(\left\{\mu_{i}: i \leqslant k\right\}, q_{k+1}\right) \in \mathbb{A}(\mathbb{M})$ and for every $H \in \mathcal{H}$, if there exists $(H, u) \leqslant\left(H, q_{k+1}(H)\right)$ with $(H, u) \in D$, then $\left(H, O T\left(q_{k+1}, H\right)\right) \in D$. This $q_{k+1}$ is produced by a finite recursion, starting from $q_{k}$, considering each of the finitely many $H \in \mathcal{H}$. For this we need two simple observations. The first observation is that by openness of $D$, if $(H, u) \in D$, then we can shrink $u$ further to make it oscillate at $H$. The second observation is that if we have found such $(H, u) \in D$, then we can add back to $u$ all $\mu_{i}$ that are not in $H$ and the trees above these from $q_{k}$ or the intermediate tree from this present finite recursion. Since $\mu_{k} \in H$ and $\mu_{k}$ is the last element of the leading set $\left\{\mu_{i}: i \leqslant k\right\}$, the part of this 
enlarged tree that oscillates at $H$ is still $u$. In the end we choose $\mu_{k+1} \in \operatorname{Split}\left(q_{k+1}\right)$ such that $\left\langle\mu_{i}: i \leqslant k+1\right\rangle$ is leading and an increasing initial segment of $\operatorname{Split}\left(q_{k+1}\right)$. Maybe for this we have to prune $q_{k+1}$ a bit further. This finishes our construction, and we let $q$ be the tree generated by $\left\{\mu_{k}: k<\omega\right\}$. It is easy to verify that $q$ is as desired.

By performing another, much simpler, fusion we can apply the Main Lemma infinitely often and obtain the following consequence:

Corollary 2.11. Let $(F, p) \in \mathbb{A}(\mathbb{M})$ and let $\left\langle D_{k}: k<\omega\right\rangle$ be a family of dense and open subsets of $\mathbb{A}(\mathbb{M})$. There exists $(F, q) \in \mathbb{A}(\mathbb{M})$ such that $(F, q) \leqslant(F, p)$ and for every $k<\omega$ the following holds:

For every $H \subseteq \operatorname{Split}(q)$ such that $(H, q) \in \mathbb{A}(\mathbb{M}),(H, q) \leqslant(F, q)$, $H$ is leading and the last element of $H$ has length at least $k$, and for every $i \leqslant k$, if there exists $\left(H, q^{\prime}\right) \in \mathbb{A}(\mathbb{M})$ such that $\left(H, q^{\prime}\right) \leqslant(H, q)$ and $\left(H, q^{\prime}\right) \in D_{i}$, then $(H, O T(q, H)) \in$ $D_{i}$.

Theorem 2.12. Forcing $\mathbb{A}(\mathbb{M})$ is proper.

Proof. Let $(N, \in)$ be a countable elementary substructure of $(H(\chi), \in)$, for some large regular cardinal $\chi$. Let $(F, p) \in \mathbb{A}(\mathbb{M}) \cap N$. Let $\left\langle D_{k}: k<\omega\right\rangle$ list all dense open subsets of $\mathbb{A}(\mathbb{M})$ that belong to $N$. Applying the proof of Corollary 2.11 (i.e. performing the obvious fusion indicated there), we obtain $(F, q) \in \mathbb{A}(\mathbb{M}),(F, q) \leqslant$ $(F, p)$, as there. We claim that this $(F, q)$ is $N$-generic for $\mathbb{A}(\mathbb{M})$. To see this, let $k<\omega$ and let $G$ be an $\mathbb{A}(\mathbb{M})$-generic filter over $\mathbb{V}$ such that $(F, q) \in G$. Let $p_{G}=\bigcup\{H: \exists u(H, u) \in G\}$ be the associated Miller tree. Note that $G$ consists of all $(H, u) \in \mathbb{A}(\mathbb{M})$ such that $H$ is an initial segment of Split $\left(p_{G}\right)$ and $p_{G} \subseteq u$. By genericity there exists $(H, u) \in G \cap D_{k}$. By Fact 2.8 there exists $\left(H^{\prime}, u^{\prime}\right) \in G$ such that the last element of $H^{\prime}$ has length at least $k, H^{\prime}$ is leading and $u^{\prime}$ oscillates at $H^{\prime}$. Without loss of generality we may assume $\left(H^{\prime}, u^{\prime}\right) \leqslant(H, u)$ and $\left(H^{\prime}, u^{\prime}\right) \leqslant$ $(F, q)$, and hence $\left(H^{\prime}, u^{\prime}\right) \in D_{k}$. As the fusion in the proof of Corollary 2.11 that produced $(F, q)$ was performed so that every initial segment of it and hence every intermediate tree was an element of $N$, at the time when we considered $H^{\prime}$ and $D_{k}$, by elementarity we had the possibility of shrinking the intermediate tree to some subtree, say $r$, so that $\left(H^{\prime}, O T\left(r\left(H^{\prime}\right), H^{\prime}\right)\right) \in D_{k} \cap N$. Therefore, by the Main Lemma, we used that possibility. Note that since $u^{\prime}$ oscillates at $H^{\prime}$ we have $\left(H^{\prime}, u^{\prime}\right) \leqslant\left(H^{\prime}, O T\left(q\left(H^{\prime}\right), H^{\prime}\right)\right)$, and $\left(H^{\prime}, O T\left(q\left(H^{\prime}\right), H^{\prime}\right)\right) \leqslant\left(H^{\prime}, O T\left(r\left(H^{\prime}\right), H^{\prime}\right)\right)$ follows by construction. We conclude that $\left(H^{\prime}, O T\left(r\left(H^{\prime}\right), H^{\prime}\right)\right) \in G \cap D_{k} \cap N$, and hence $(F, q)$ is $N$-generic for $\mathbb{A}(\mathbb{M})$ as claimed.

Remark 2.13. Main Lemma 2.10 can be used to show that $\mathbb{A}(\mathbb{M})$ satifies Baumgartner's Axiom $A$ (a slightly stronger property than properness).

By Fact 2.4 and Theorem 2.12 we obtain:

Corollary 2.14. Every finite power of $\mathbb{M}$ is proper; similarly for $\mathbb{L}$.

We can as well mix $\mathbb{L}$ and $\mathbb{M}$ as follows: Let $\omega^{\prime}$ be a disjoint isomorphic copy of $\omega$ and let $\mathbb{M}^{\prime}$ be the Miller forcing defined on $\omega^{\prime}$. We define a mixed amoeba forcing $\mathbb{A}\left(\mathbb{L} \cup \mathbb{M}^{\prime}\right)$ as follows. The conditions are $\left(F \cup H^{\prime}, p \cup q^{\prime}\right)$ such that $(F, p) \in \mathbb{A}(\mathbb{L})$ and $\left(H^{\prime}, q^{\prime}\right) \in \mathbb{A}\left(\mathbb{M}^{\prime}\right)$. The order is the natural amoeba order. We can carry over all our definitions and results to $\mathbb{A}\left(\mathbb{L} \cup \mathbb{M}^{\prime}\right)$ and in particular obtain: 
Theorem 2.15. Forcing $\mathbb{A}\left(\mathbb{L} \cup \mathbb{M}^{\prime}\right)$ is proper.

Since every forcing of the form $\mathbb{L}^{n} \times \mathbb{M}^{m}$ completely embeds into $\mathbb{A}\left(\mathbb{L} \cup \mathbb{M}^{\prime}\right)$, we obtain:

Theorem 2.16. Every finite product of the form $\mathbb{L}^{n} \times \mathbb{M}^{m}$ is a complete subforcing of $\mathbb{A}\left(\mathbb{L} \cup \mathbb{M}^{\prime}\right)$ and hence proper.

Remark 2.17. Suppose that $p_{G} \in \mathbb{M}$ is $\mathbb{A}(\mathbb{M})$-generic. Bagaria and Di Prisco have noticed that by our [8, Main Lemma 2.2] it follows that every pair $(x, y) \in\left[p_{G}\right] \times\left[p_{G}\right]$ with $x \neq y$ is $\mathbb{M}^{2}$-generic. Clearly this is false for $\mathbb{L}$ in place of $\mathbb{M}$, as every $\mathbb{L}^{2}$ generic pair $(x, y)$ has the property that $\langle\operatorname{sign}(x(n)-y(n)): n\langle\omega\rangle$ is a Cohen real. Thus the problem is raised to characterize those $n$-tuples in $\left[p_{G}\right]$ that are $\mathbb{M}^{n}$-generic, and similarly for $\mathbb{L}$. I am grateful to the referee for detecting an error in an argument on this question in a previous version of this paper.

Remark 2.18. Using the main ideas of this paper, one can show properness of $\mathbb{L}^{n}, \mathbb{M}^{n}$ directly. This proof actually shows that $\mathbb{L}^{n}, \mathbb{M}^{n}$ satisfy Axiom A.

\section{REFERENCES}

1. J. E. Baumgartner, Iterated forcing, surveys in set theory, London Mathematical Society Lecture Note Series, no. 87 (A.R.D. Mathias, ed.), Cambridge University Press, Cambridge, 1983, pp. 1-59. MR823775 (87c:03099)

2. J. Brendle, Mutual generics and perfect free subsets, Acta Math. Hungar. 82 (1999), pp. 143-161. MR1658566 (2000g:03117)

3. R. Laver, On the consistency of Borel's conjecture, Acta Math. 137 (1976), pp. 151-169. MR0422027 (54:10019)

4. A. Miller, Rational perfect set forcing, Contemporary Mathematics, 31, Amer. Math. Soc., Providence, RI, 1984, pp. 143-159. MR763899 (86f:03084)

5. S. Shelah, Proper forcing, Lecture Notes in Mathematics, vol. 940, Springer-Verlag, Berlin-New York, 1982. MR675955 (84h:03002)

6. S. Shelah and O. Spinas, The distributivity numbers of finite products of $\mathcal{P}(\omega) /$ fin, Fundamenta Mathematicae 158 (1998), pp. 81-93. MR.1641157 (2000b:03170)

7. O. Spinas, Generic trees, Journal of Symbolic Logic 60 (1995), pp. 705-726. MR.1348990 (96g:03087)

8. O. Spinas, Ramsey and freeness properties of Polish planes, Proceedings of the London Mathematical Society (3) 82 (2001), no. 1, pp. 31-63. MR.1794256 (2001j:03088)

9. B. Velickovic and H. Woodin, Complexity of reals in inner models of set theory, Ann. Pure Appl. Logic 92 (1998), pp. 283-295. MR:1640916 (99f:03067)

Mathematisches Seminar der Christian-Albrechts-Universität Zu Kiel, LudewigMeyn-Strasse 4, 24098 Kiel, Germany

E-mail address: spinas@math.uni-kiel.de 